\title{
Weather-induced ischemia and arrhythmia in patients undergoing cardiac rehabilitation: another difference between men and women
}

\author{
Alexandra Schneider • Angela Schuh • \\ Friedrich-Karl Maetzel • Regina Rückerl • \\ Susanne Breitner • Annette Peters
}

Published online: 26 April 2008

(C) ISB 2008

Erratum to: Int $\mathbf{J}$ Biometeorol

DOI 10.1007/s00484-008-0144-9

Unfortunately an error occurred in Table 8: In the column of men there should be "Odds ratio" instead of "p-value".

The online version of the original article can be found at http://dx.doi. org/10.1007/s00484-008-0144-9.

A. Schneider $(\bowtie) \cdot$ R. Rückerl $\cdot$ S. Breitner $\cdot$ A. Peters

Helmholtz Zentrum München,

German Research Center for Environmental Health, Institute of Epidemiology,

Neuherberg, Germany

e-mail: alexandra.schneider@helmholtz-muenchen.de

\begin{abstract}
A. Schneider $\cdot$ A. Schuh Institute of Health and Rehabilitation Sciences,

Ludwig-Maximilians-University of Munich,

Munich, Germany
\end{abstract}

F.-K. Maetzel

Curschmann Clinic,

Timmendorfer Strand, Germany 